cases. All instances of self limiting liver injury not requiring admission would not have been detected. Severe liver disorders, on the other hand, may occur without early changes in standard liver function. ${ }^{18}$

Acute liver injury associated with use of nonsteroidal anti-inflammatory drugs is considered to be the result of current rather than past exposure. Therefore we chose as the comparison group the same people who had taken non-steroidal anti-inflammatory drugs at a time when they were no longer taking the drugs. ${ }^{19}$ We compared the incidence rate of acute liver injury during the total person time when subjects were taking non-steroidal anti-inflammatory drugs with the incidence rate during the total person time when they were not. The use of the exposed population as its own comparison group contributes to a reduction in confounding. Risk characteristics that do not change with time will necessarily be the same for exposed and nonexposed patients. We must, however, consider the possibility of changes in the use of non-steroidal antiinflammatory drugs when applying this kind of cross over study design. The decision to start or stop use may be influenced by the presence of prodromal symptoms of the acute liver injury. We could not directly assess the extent of bias related to the beginning of medication for early non-specific symptoms of liver injury not yet diagnosed. For the 12 current users, however, for whom information on the indication for use of nonsteroidal anti-inflammatory drugs was recorded, osteoarthritis was the underlying clinical condition. We evaluated the pattern of stopping treatment. Only four out of the 18 patients in the group not currently taking non-steroidal anti-inflammatory drugs had filled a prescription for non-steroidal anti-inflammatory drugs two to six months before admission to hospital; four had filled one prescription seven to 12 months before admission; and the remaining 10 had not filled a prescription in the year before admission. Increasing the criterion for current use of non-steroidal anti-inflammatory drugs to up to 90 days after the prescription did not materially change the rate ratio estimate.

The effects if non-steroidal anti-inflammatory drugs can be seen most clearly in a population free of the "background noise" of hepatotoxicity induced by other drugs. ${ }^{20}$ In the subpopulation with no use of other hepatotoxic drugs the rate ratio estimate of the effect of non-steroidal anti-inflammatory drugs on acute liver injury was larger than in the overall population.

Some of the patients did not undergo tests to rule out infectious hepatitis. In addition, diagnostic tests for hepatitis $C$ were not available during the study, and differential diagnosis for cholelithiasis was not always carefully considered. All these limitations must be considered when interpreting our results, and some cases may have been misclassified. Any misclassifica- tion, however, would be similar in the two groups and may therefore have resulted in some underestimation of the true relative risk. Given the low incidence of acute liver injury, the impact on the excess risk would have been small.

In summary, our results show that admissions to hospital for acute non-infectious liver injury are rare and that current use of non-steroidal anti-inflammatory drugs is associated with a twofold increase in the risk and an excess risk of five per 100000 person years.

We thank Edith Malcolm and the staff of the pharmacoepidemiology unit in Saskatchewan for their expert collaboration with the Saskatchewan health databases and the organisation of the medical record abstraction process and Drs David Henry (University of Newcastle), Hershel Jick (Boston University), and Bruno Stricker (Netherlands cente for monitoring of adverse reactions to drugs), who read earlie versions of the manuscript.

This study was supported by a grant from CIBA-GEIGY AG, Basel. It is based in part on data provided by the Saskatchewan department of health. The interpretation and conclusions contained herein do not necessarily represent those of the government of Saskatchewan or the Saskatchewan department of health

1 Lewis $\mathrm{JH}$. Hepatic toxicity of nonsteroidal anti-inflammatory drug. Clin Pharm 1984;3:128-38.

2 Beard K, Belic L, Aselton P, Perera D, Jick H. Outpatient drug-induce parenchymal liver disease requiring hospitalization. $\mathrm{f}$ Clin Pharmacol 1986;26:633-7.

3 Jick H, Derby LE, Garcia Rodriguez LA, Jick SS, Dean AD. Liver disease associated with diclofenac, naproxen and piroxicam. Pharmacotherapy 1992;12:207-12.

4 Strand LM, West R. The health databases in Saskatchewan. In: Strom B, ed Pharmacoepidemiology. New York: Churchill Livingstone, 1989:189-200.

5 Garcia Rodriguez LA, Walker AM, Pérez Gutthann S. Non-steroidal antiinflammatory drugs and gastrointestinal hospitalizations in Saskatchewan a cohort study. Epidemiology 1992;3:337-42.

6 Sherlock S, ed. Diseases of the liver and biliary system. Oxford: Blackwell, 1981.

7 Biorneboe M, Iversen O, Olsen S. Infective hepatitis and toxic jaundice in a municipal hospital during a five-year period. Acta Med Scand 1967;182:491-
mune 501 .

8 Ross RM. Hepatic dysfunction secondary to heart failure. Am $\mathcal{f}$ Gastroenter 1981;76:511-8.

9 Raviolo P, Rizzetto M, Tabone M. Colestasi intraepatica in corso ipertiroidismo. Recenti Prog Med 1991;82:319-23.

10 Gomar Sancho C. Anestesia y alteraciones hepáticas postoperatorias. Med Clin 1988;90:579-82.

11 Report of an international consensus meeting. Criteria of drug-induced live disorders. I Hepatol 1990;11:272-6.

12 Stricker BH, Spoelstra P, eds. Drug-induced hepatic injury. Amsterdam: Elsevier, 1985

13 Walker AM, ed. Observation and inference: an introduction to the methods of epidemiology. Newton: Epidemiology Resources Inc, 1991.

14 Martin D, Austin $H$. An efficient program for computing condition maximum likelihood and exact confidence limits for a common odds ratio. Epidemiol 1991;2:359-62.

15 Pessayre D, Larrey D. Acute and chronic drug-induced hepatitis. Baillieres Clin Gastroenterol 1988;2:385-422.

16 Van Ness MM, Diehl AM. Is liver biopsy useful in the evaluation of patient with chronically elevated liver enzymes? Ann Intern Med 1989;111:473-8.

17 Zimmerman HJ. Effects of aspirin and acetaminophen on the liver. Arch Inter Med 1981;141:333-42.

18 Davis M. Drugs and abnormal liver function tests. Adv Drug Reac Bul 1989;139:520-3

19 Ray WA, Griffin MR. Use of medicaid data for pharmacoepidemiology. Am f Epidemiol 1989;129:837-49.

20 Rothman $\mathrm{KJ}$, Poole $\mathrm{C}$ A strengthening programme for weak associations. Int F Epidemiol 1988;17:955-9.

(Accepted 30 fuly 1992)

\section{Generalised pustular psoriasis: response to topical calcipotriol}

\section{J Berth-Jones, J Bourke, K Bailey, R A C Graham-Brown, P E Hutchinson}

Pustular psoriasis is a life threatening disease ${ }^{1}$ and usually requires treatment with systemic drugs which themselves carry significant risks. Calcipotriol, a vitamin $\mathrm{D}$ analogue, is an effective new topical treatment for psoriasis vulgaris. ${ }^{2}$ This is the first report of its use in pustular psoriasis.

\section{Case reports}

Three consecutive patients admitted with pustula psoriasis were treated with calcipotriol ointment 50 $\mu \mathrm{g} / \mathrm{g}$ (Dovonex).

Case 1-A 68 year old woman had been controlling her psoriasis using tar and betamethasone valerate ointment until three weeks before admission. Her skin then deteriorated until $45 \%$ of her body surface was affected by erythema and pustulation. Calcipotriol ointment was applied twice daily. Within 24 hours pustulation had completely resolved. She continued to improve and was discharged after three weeks to continue using the same treatment. In total, $300 \mathrm{~g}$ calcipotriol ointment was applied during the admission.
Correspondence to: 
Department of Dermatology, Leicester

Royal Infirmary, Leicester LE1 5 WW

J Berth-Jones, senior registrar in dermatology $\mathrm{J}$ Bourke, registrar in dermatology

K Bailey, house physician R A C Graham-Brown, consultant in dermatology P E Hutchinson, consultant in dermatology
Case 2-A 76 year old woman had a history of two previous admissions with less severe episodes of generalised pustular psoriasis. Treatment with methotrexate and prednisolone was required on the first occasion and with both these drugs and etretinate on the second. Her condition had been adequately controlled for two years with etretinate $25 \mathrm{mg}$ daily. Then, over three weeks, her condition rapidly deteriorated. Ninety per cent of her skin surface was erythematous and covered in sheets of pustules. She was not eating or drinking and required an intravenous infusion. Serum biochemical studies showed severe impairment of renal and hepatic function, increasing the risks of systemic treatment. After three days of bed rest and treatment with emollients she was feverish and increasingly weak. Calcipotriol ointment was then applied twice daily and within 24 hours the pustulation had completely resolved. After 10 days, during which about $300 \mathrm{~g}$ ointment was used, both the erythema and her general condition had greatly improved. Etretinate was then recommenced at a dose of $50 \mathrm{mg} / \mathrm{day}$ and she was discharged two weeks later to continue this treatment

Case 3-An 81 year old woman was admitted with pustular psoriasis in the flexures and extending to the limbs and trunk, so that some $30 \%$ of the body surface was affected. Calcipotriol ointment was applied twice daily at a rate of $15 \mathrm{~g} /$ day. White soft paraffin was used liberally. Within 48 hours the pustulation had completely resolved. One week later the rash had cleared, treatment was discontinued, and the patient was observed. After an interval of seven days a minor recurrence of the eruption developed. This also responded promptly to topical calcipotriol but we decided to use oral methotrexate $5 \mathrm{mg} /$ week to prevent any further relapse.

\section{Comment}

Bed rest and emollients are undoubtedly beneficial in generalised pustular psoriasis. However, these seem unlikely to be entirely responsible for the improvement seen in our patients, since all three improved so promptly on treatment with calcipotriol. In case 2 it was very clear that the patient was growing increasingly weak and toxic after admission. Not only the pustulation but also her general condition improved rapidly with calcipotriol. Previous, less severe episodes in this patient had required far more aggressive treatment.

Irritant reactions are often seen with calcipotriol but $\tau$ did not present a problem in these cases. A more important potential hazard is hypercalcaemia. ${ }^{3}$ For $\bar{c}$ chronic plaque psoriasis calcipotriol ointment has been used in large studies in quantities of up to $100 \mathrm{~g}$ weekly without any evident effect on calcium homoeostasis. ${ }^{2}$ No data are available for other forms of the disease. The serum calcium concentration was therefore carefully monitored in each case and did not rise.

Calcipotriol may prove to be a new, effective, and relatively safe treatment for generalised pustular psoriasis. However, we emphasise the need for daily monitoring of the serum calcium value. This form of psoriasis remains a life threatening condition and will always require management in specialist dermatological units because of the accompanying severe metabolic complications and the risk of septicaemia.

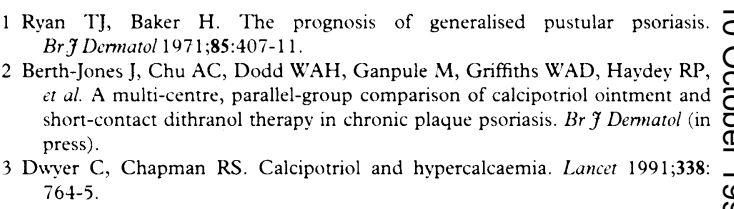

\section{A PATIENT WHO CHANGED MY PRACTICE}

\section{Challenge of sarcoid heart disease}

Paul Wood once light heartedly defined a physician as "a chap who is prepared to diagnose a condition that he did not know existed"-an exacting definition that would exclude many of us. I was humbled to learn that a patient I had seen was shown at necropsy to have sarcoid heart disease, a condition that I was aware of, though I had never then diagnosed it, thought that I was quite prepared to do so, but had not even considered it in her case. In partial exoneration was the fact that she had never been "my patient," but I had been asked to see her because of her rhythm problems. after a dilatation and curettage eight years before her death. She subsequently suffered from supraventricular tachycardia, left bundle branch block, partial heart block, transient complete heart block, and multifocal ventricular ectopic beats before reverting to sinus rhythm before her sudden death at the age of 59 in 1968.

She was the first of six cases of sarcoid heart disease that we published in 1972. I had always been dissatisfied with the dead end diagnosis of "cardiomyopathy" and these cases made me realise that we must strive to be more precise in refining the diagnosis and also that sarcoid of the heart might be more common than we, or the literature, had previously thought.

This led me to canvass my colleagues for further cases. Many confessed that they had never even entertained the diagnosis. Soon we had a considerable number of cases on the files, always a disproportionate number from East Anglia. By 1974 we published 50 cases; by 1981, 197 cases; and by 1986 over 300 cases in the United Kingdom.

Before this study there was a single case report from the United Kingdom and before many years had passed our series probably outnumbered the entire reported world experience at that time.

This intriguing disease with so many bizarre and puzzling manifestations fascinated me with its endles variety and challenges. We followed up all the cases and the resultant database was extensively consulted by colleagues in many parts of the world, and they added their own cases to the series. The many problems of diagnosis and management became better understood though the mysteries of causation and occurrence, and indeed the aetiology of sarcoidosis itself, remain to be solved.

At least the problem has been brought out into the open, the need for recognition has been exposed, and several lives have been saved as a result. If only I had been more aware in 1960 we might have managed to save the first patient.

An incidental spin off has been the number of international friendships that have resulted and the opportunities to lecture or give papers in 10 countries over the years.-HUGH A FLEMING, emeritus consultant cardiologist, Cambridge

Ghosh P, Fleming HA, Gresham GA, Stovin PGI. Myocardial sarcoidosis. Br Heart f 1972;34:769-73.

Fleming HA. Death from sarcoid heart disease. United Kingdom series 1971-86. 300 cases with 138 deaths. In: Grassi C, et al, eds. Sarcoidosis and other granulomatous disorders. Amsterdam:Elsevier Science Publications, 1988:19-33. 